\title{
Optimal resource allocation for LTE uplink scheduling in smart grid communications
}

\author{
Jian Li, Yifeng He, Yun Tie, Ling Guan \\ Department of Electrical and Computer Engineering, Ryerson University, Toronto, Canada \\ Email address: \\ jian.li@ryerson.ca(J. Li), yhe@ee.ryerson.ca(Y. He), ytie@ee.ryerson.ca(Y. Tie), lguan@ee.ryerson.ca(L. Guan)
}

To cite this article:

Jian Li, Yifeng He, Yun Tie, Ling Guan. Optimal Resource Allocation for LTE Uplink Scheduling in Smart Grid Communications. International Journal of Wireless Communications and Mobile Computing. Vol. 1, No. 4, 2013, pp.113-118.

doi: 10.11648/j.wcmc.20130104.15

\begin{abstract}
The success of the smart grid majorly depends on the advanced communication architectures. An advanced smart grid network should satisfy the future demands of the electric systems in terms of reliability and latency. The latest 4th-generation (4G) wireless technology, the 3rd Generation Partnership Project (3GPP) Long Term Evolution (LTE), is a promising choice for smart grid wide area networks (WAN), due to its higher data rates, lower latency and larger coverage. However, LTE is not a dedicated technology invented for smart grid, and it does not provide Quality of Service (QoS) guarantee to the smart grid applications. In this paper, we propose an optimal LTE uplink scheduling scheme to provide scheduling timeguarantee at the LTE base station for different class of traffic, with a minimal number of total resource blocks. A lightweight heuristic algorithm is proposed to obtain the optimal allocation of resource blocks for each class of traffic. In the simulation, we compare the proposed optimal scheduling scheme and two existing scheduling schemes, the Large-Metric-First scheduling scheme and the Guaranteed Bit Rate (GBR) /Non-GBR scheduling scheme. The comparison results demonstrate that the proposed optimal scheduling can use less resource blocks to satisfy the scheduling time requirements than the other two existing scheduling schemes.
\end{abstract}

Keywords: LTE, Uplink, Scheduling, Smart Grid Communications, Quality of Service

\section{Introduction}

The smart grid is a modern electric system which uses sensors, automation, computers and other application-specific devices to control and monitor the grid system. Currently, the constant improvements of smart grid technology have made a significant progress on flexibility, security, reliability and efficiency of the electricity system. Meanwhile, the advanced systems and devices generate a largevolume of traffic flows and placeanenormous challenge on real-time communications. Therefore, an advanced and efficient smart grid communication network should be able to satisfy the future demands in terms of reliability and latency. Smart grid communication architecture consists of three interconnected networks: Wide Area Network (WAN), Neighborhood Area Network (NAN) and Home Area Network (HAN) [1]. The WAN plays animportant role in the smart grid communication network, which connects various NANs, and forms a connected, integrated and robust smart grid system. The performance of WAN directly affects the systemmonitoring and controlling, or even whole electric system operations. In this paper, we study the uplink scheduling problem in the smart grid WAN.

In many countries, data fiber network, synchronous optical network (SONET), supervisory control and data acquisition (SCADA) network, etc., are being deployed in current smart grid WANs [2]. However, the smart grid WAN is still unsatisfactory in some aspects, such as lack of common backhaul medium for data communication. Furthermore, future demands including Plug-in Hybrid Electric Vehicle (PHEV) and Community Energy Storage (CES) require reliable two-way communications and interactivities that traditional network systems cannot provide $[3,4]$. Therefore, the traditional communication architecture should be urgently upgraded, or even replaced by a more advanced communication technology.

There are several kinds of technologies available for smart grid WAN, such as Long Term Evolution (LTE), Worldwide Interoperability for Microwave Access (WiMAX) [5], etc.Compared with other technologies, the latest 4th-generation $(4 \mathrm{G})$ wireless technology, the 3rd Generation Partnership Project (3GPP) LTE is a promising 
option for smart grid because of its higher data rates, lower latency and larger coverage. The 3GPP LTE Release 8 shows that LTE provides up to $300 \mathrm{Mbps}$ download rate and $75 \mathrm{Mbps}$ upload rate [6]. The specification also defines Orthogonal Frequency Division Multiple Access (OFDMA) as the access technique for the downlink and Single Carrier FDMA (SC-FDMA) for the uplink. OFDMA has advantages of robustness against multi-path fading, higher spectral efficiency and bandwidth scalability; and SC-FDMA makes user equipments (UEs) energy-saving. The additional crucial technique applied in LTE is Multiple-Input-Multiple-Output (MIMO) that uses multiple transmitters and receivers to achieve higher bit rates and improved coverage [7]. Fig.1 shows the smart grid connectivity supported by LTE.

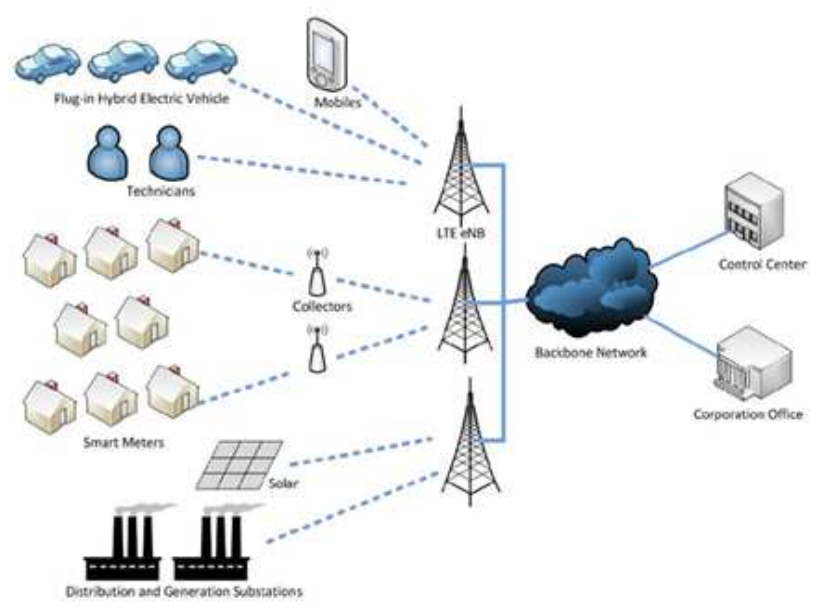

Figure 1.The smart grid connectivity supported by LTE

However, LTE is not a dedicated technology invented for smart grid. Smart grid applications have outstanding Quality of Service (QoS) requirements. Smart grid applications need more stringent latency requirements in WAN than other public applications such as web. An excessive delay of the criticaldata may delay the power restoration, which may lead to severe economic and social consequences. Reducing latency or end-to-end delay becomes one of the major challenges in smart grid communication networks.

In this paper, we investigate the LTE uplink scheduling problem in a smart grid WAN. Ourcontribution is that we optimize the allocation of resource blocks (RBs) in the LTE base station, named evolved NodeB (eNB) in LTE, to provide scheduling timeguarantee to different classes of smart grid traffic.

This paper is organized as follows. We describe the related works in Section 2. In Section 3, we study a queuing model and an LTE uplink scheduling model. Based on the models, we theoretically analyze the relationship between the scheduling time and the resource blocks to be allocated. Section 4 presents the problem formulation and heuristic algorithm forthe LTE uplink scheduler. The experiment results are presentedin Section 5, and the conclusions are drawn in Section 6.

\section{Related Work}

Since the LTE technical standard does not define a unique scheduling algorithm, the LTE scheduling has attracted significant attention from researchers. In [8], YuzheXu et al. investigated the latency performance of LTE network in smart grid, and proposed a new LTE scheduler that firstly allocates resources for smart grid. In [9], Yasir et al. presented a Bandwidth and QoS Aware (BQA) LTE uplink scheduler, which maximizes the cell throughput by giving priority to user equipments (UEs) with better channel conditions and maintains some level of fairness by providing resources to UEs with adverse channel conditions. In [10], Oscar et al.proposed two novel LTE uplink resource allocation algorithms for multiclass services, which adapt dynamically to the number of requests in the system, assigning resources as fair as possible.

\section{System Models}

We study a queuing model and LTE uplink scheduling model in this section. The queuing model illustrates a method to obtain the scheduling time for the LTE uplink scheduler. Section 3.2 shows the LTE uplink scheduling model, which presents the mechanism of LTE uplink scheduling and the relationship between the scheduling time and LTE scheduling resources.

\subsection{Queuing Model}

According to the requirements, the data in the smart grid can be classified into different classes. For example, data on remote workforce is classified into low-priority class while the control data from the control center and exception messages such as the outage notifications are classified into the critical class. The queuing model consisting of several queues and one scheduler is involved in our study. The queuing model in LTE scheduler is shown in Fig.2. Suppose that the scheduler provides $C$ classes of traffic with different priorities. A smaller classnumber corresponds to a higher priority. The traffic of class- $c(\forall c=1,2, \ldots, C)$ is characterized by four parameters: 1) the arrivals of the class-crequests, which are modeled as a Poisson process with average arrival rate $\lambda_{c}$ requests/second; 2) the average request $\operatorname{size} F_{c} \mathrm{Kbytes} /$ request which is specified by the size of every request; 3 ) the upper bound of scheduling time $\tau_{c}$ in seconds; and 4$)$ the possibility $\rho_{c}$ that an arriving request belongs to class- $c$.

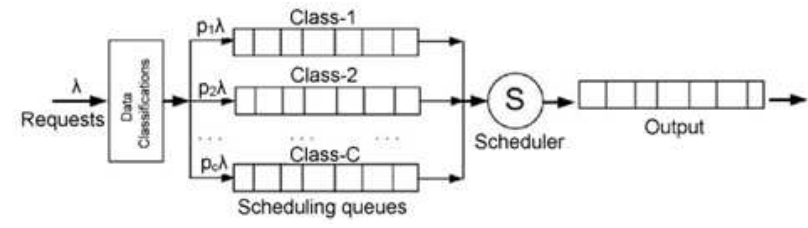

Figure 2.Queuing model 
In order to simplify the queuing model, we assume that the model consists of $C$ queues connecting to a scheduler, and each queueis used to hold the traffic of the corresponding class. Requests can be served immediately at the scheduling rate $S_{\text {total }}$ by the scheduler. In this paper, we employ the preemptive priority service scheme.

In the queuing model, the scheduling rate for class-cis denoted as $S^{(c)}$. We have $S_{\text {total }}=\sum_{c=1}^{C} S^{(c)}$. The average size of requests of class- $c$ is $F_{c}$. Thus, the scheduling time for class-ctraffic flows is assumed to be Poisson distributed with a mean timeof $F_{c} / S^{(c)}$. In accordance with the composition property of the Poisson process, the arrivals of task requests in class- $c$ follow a Poisson process with arrival rate $\lambda_{c}=\rho_{c} \lambda$ and the total arrivals of all requests follow a Poisson process with average arrival rate $\lambda=\sum_{c=1}^{C} \lambda_{c}$. In a preemptive priority $\mathrm{M} / \mathrm{M} / 1$ queuing system, the mean scheduling time for class- $c$ data flowis given by[11]:

$$
T_{s c h}^{(c)}=\frac{F_{c} / S^{(c)}}{1-\beta^{(c-1)}}+\frac{\sum_{j=1}^{c}\left(\rho_{c} \lambda F_{j}^{2} / S^{(j)^{2}}\right)}{\left(1-\beta^{(c-1)}\right)\left(1-\beta^{(c)}\right)}
$$

where $\beta^{(c)}=\sum_{j=1}^{c}\left(\rho_{j} \lambda F_{j}\right) /\left(S^{(j)}\right)$. To ensure the schedule queue stable, $\beta^{(C)}=\sum_{j=1}^{C}\left(\rho_{j} \lambda F_{j}\right) /\left(S^{(j)}\right)<1$ should be satisfied.

\subsection{LTE Uplink Scheduling Model}

The LTE uplink scheduleris located at the base station in LTE. The minimum transmission unit of LTE scheduleris known as a resource block. The radio resource that is available in the uplink LTE system is defined in both frequency and time domains. In the frequency domain, each $\mathrm{RB}$ consists of 12 consecutive subcarriers, and in the time domain it is made up of one time slot of $0.5 \mathrm{~ms}$ duration. Each $1 \mathrm{~ms}$ Transmission Time Interval (TTI) consists of 2 slots, and eachsub-frame is defined as 10 TTIs. At each TTI, multiple RBs can be assigned to a number of users with different classes; each resource block, however can be assigned to at most one user.

The LTE scheduler has $B \mathrm{MHz}$ bandwidth, divided into $N$ RBs. We assume that the scheduler is capable of assigning RBs arbitrarily to all users, and each RB $n$ has a bandwidth of $B / N$. Let $n=\{1,2 \ldots, N\}$ denotes the RB indexset. For simplicity, we suppose that uniform power allocation across all subcarrier.

We define a variable $x_{c}$ to indicate the number of resource blocks assigned to class- $c$ traffic flows. Assuming that the throughput usage can achieve the Shannon rate limit [12], the maximum uplink channel throughput of class- $c$ for uplink direction according to Shannon-Hartley theory can be expressed as [13]:

$$
S^{(c)}=x_{c} \frac{B}{N} \log _{2}\left(1+\operatorname{SINR}_{c, n}\right)
$$

where $S I N R_{c, n}$ is the average Signal to Interference and Noise Ratio (SINR) for the RB $n$ at the transmitter. The LTE standard provides reporting mechanisms (Channel State Information and Buffer Status Reporting) to providethe packet scheduler with valuable information about the cellular environment that can assist in increasing the scheduling operation in the uplink [14].

Therefore, the total scheduling rate for the LTE uplink scheduling is given by:

$$
S_{\text {total }}=\sum_{c=1}^{C} x_{c} \frac{B}{N} \log _{2}\left(1+S I N R_{c, n}\right)
$$

Based on the above analysis, we can formulate the mean scheduling time for processing class-ctraffic as follows:

$$
\begin{gathered}
T^{(c)}=\frac{F_{c} /\left(x_{c} \frac{B}{N} \log _{2}\left(1+\operatorname{SINR}_{c, n}\right)\right)}{1-\sum_{j=1}^{c-1} \frac{\lambda_{j} F_{j}}{x_{j} \frac{B}{N} \log _{2}\left(1+S I N R_{j, n}\right)}}+ \\
\frac{\sum_{j=1}^{c}\left(\lambda_{j} F_{j}^{2} /\left(x_{j} \frac{B}{N} \log _{2}\left(1+\operatorname{SIN} R_{c, n}\right)\right)^{2}\right)}{1-\sum_{j=1}^{c-1} \frac{\lambda_{j} F_{j}}{x_{j} \frac{B}{N} \log _{2}\left(1+\operatorname{SINR} R_{j, n}\right)}} \\
\frac{1}{1-\sum_{j=1}^{c} \frac{\lambda_{j} F_{j}}{x_{j} \frac{B}{N} \log _{2}\left(1+S I N R_{j, n}\right)}} \\
\forall c=1,2, \ldots, C,
\end{gathered}
$$

The totalschedulingtime for processing all requests is formulated as follows:

$$
T_{\text {total }}=\sum_{c=1}^{C} \frac{\lambda_{c}}{\lambda} T^{(c)}
$$

\section{Optimal Resource Allocation}

In this section, we formulate the resource allocation minimization problem depend on the queuing model and LTE uplink scheduling model, and propose a heuristic scheme to obtain the solution of the problem.

\subsection{Problem Formulation}

We formulate the allocated resource blocks minimization problem based on the queuing model and the LTE uplink scheduling model, aiming to minimize the total number of the allocated resource blocks while satisfying the scheduling time constraint for each class of traffic. The problem of resourceblock minimization can be written as follows:

$$
\operatorname{Maximize}_{\left\{x_{c}\right\}} x_{c}
$$

Subject to

$$
\begin{gathered}
x_{c} \leq N, \quad \forall c=1,2, \ldots, C, \\
\sum_{c=1}^{C} x_{c} \leq N, \\
\sum_{j=1}^{c} \frac{\rho_{j} \lambda F_{j}}{S^{(c-1)}}<1, \\
\lambda_{c} F_{c}<S^{(c)}, \quad \forall c=1,2, \ldots, C, \\
T^{(c)}<\tau_{c,} \quad \forall c=1,2, \ldots, C,
\end{gathered}
$$

where $(6 \mathrm{~b})$ indicates that the resource blocks assigned to class- $c$ must be less than or equal to the total number of 
resource blocks. Equation (6c) declares that the number of allocated resourceblock also cannot beyond the total number of resource blocks. Equation (6d) presents the inequality to ensure the queuing model stability. Equation (6e) shows the relationship between the arrival rate and the scheduling rate for class-c. $\tau_{c}$ in $(6 \mathrm{f})$ is the upper bound of the scheduling time for class- $c$ service. It is pre-defined according to the QoS requirements for different classes.

\subsection{Heuristic Scheme}

Although global searching to find the solution of the minimum resource blocks could be feasible, such method is inefficient. Therefore, we propose a heuristic scheme to obtain the optimal solution.

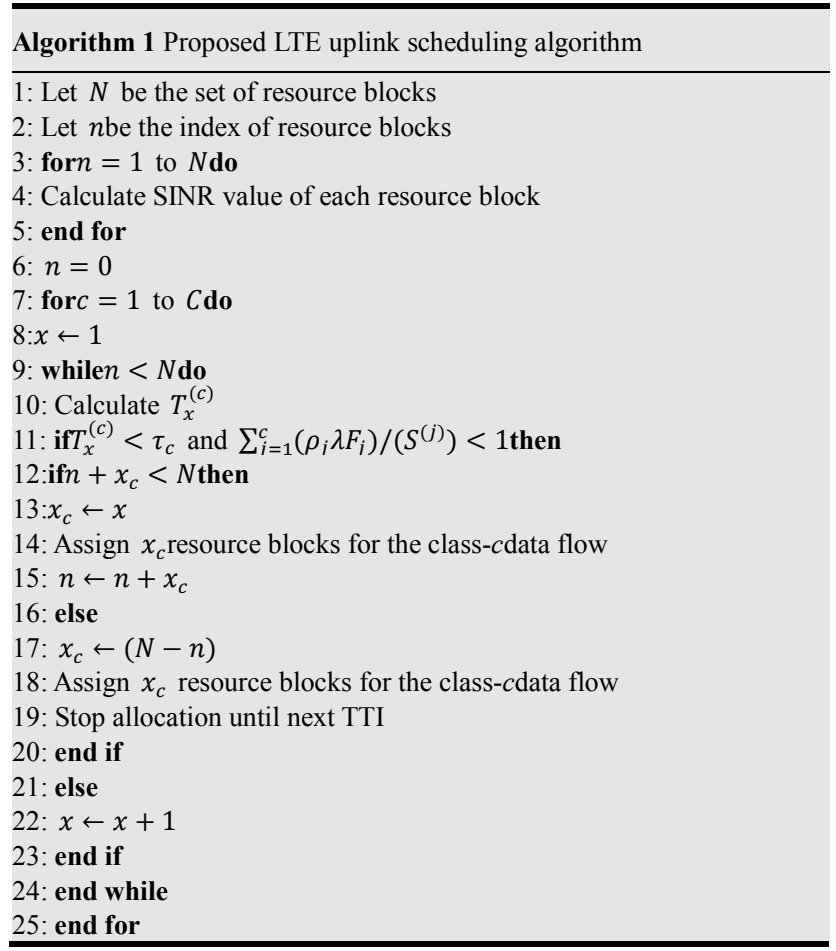

The proposed heuristic scheme dynamically decides the number of resource blocks allocated to each class in each TTI. Algorithm 1 describes the proposed heuristic scheme in details. In each TTI, the eNBscheduler captures the Buffer Status Report and Channel State Information from the user equipments and calculates SINR values. The principle of the allocation is to assign RBs in sequential order from the higher-priority classes to lower-priority classes. The initial number of resource blocks to be allocated for class-c $x$ is set to 1 . Next, the scheduler calculates the scheduling time $T_{x}^{(c)}$ using (4) and compares it with the scheduling time requirement $\tau_{c}$. If the value of calculation is larger than the requirement, the value $x$ is increased by 1 . The scheduler keeps increasing the resource blocks, until the scheduling time $T_{x}^{(c)}$ meet the requirement. When the calculated value becomes smaller than $\tau_{c}, x_{c}$ is determined and the scheduler allocates $x_{c}$ resource blocks to the class- $c$ traffic flows. Then the scheduler begins to process the next classtraffic flows. Finally, once the allocationis performed, the system updates all the relevant parameters.

Note that the system adjusts itself in order to match the QoS target. The proposed allocation scheme aims to allocate minimum RBs. Our proposed heuristic can guarantee each class of traffic is allocated the minimum RBs. If any of the class obtains one less resourceblock, this class of traffic cannot satisfy the scheduling time requirements.

\section{Simulations}

In this section, we perform LTE uplink scheduling heuristic algorithm simulations to evaluate the networkperformance in terms of the resources allocation and the scheduling time.

\subsection{Simulation Setting}

Table 1 summarizes the parameter settings of smart grid traffic. All traffic is divided into three classes. Class- 1 traffic has the highest priority, including the exception messages and alarms. Class- 2 contains the control messages which is not as critical as class-1. The normal operation traffic is classified into class-3. The total arrival rate of the incoming trafficis set in the range of 100-300 requests/second. The other simulation configurations can be seen in Table 2 . We evaluate the number of RBs allocated for different classes in different scheduling algorithms as well as the performance of the algorithms in terms of scheduling time.

Table 1.Parameter Settings of Smart Grid Traffic

\begin{tabular}{lccc}
\hline Service class & $\mathbf{1}$ & $\mathbf{2}$ & $\mathbf{3}$ \\
\hline Percentage of arrival rate & $20 \%$ & $30 \%$ & $50 \%$ \\
Average requests size (bytes) & $30 \mathrm{k}$ & $50 \mathrm{k}$ & $700 \mathrm{k}$ \\
Upper bound of scheduling time (sec) & 0.001 & 0.003 & 0.007 \\
\hline
\end{tabular}

Table 2. Major Simulation Parameters of LTE

\begin{tabular}{ll}
\hline Parameter & Setting \\
\hline System bandwidth & $10 \mathrm{MHz}$ \\
Number of RBs & 50 \\
Number of subcarriers per RB & 12 \\
RB bandwidth & $180 \mathrm{kHz}$ \\
Transmission time interval & $1 \mathrm{~ms}$ \\
Transmission power & $125 \mathrm{~mW}$ \\
Noise power per Hz & $160 \mathrm{dBm}$ \\
Traffic arrival model & Poisson \\
\hline
\end{tabular}

\subsubsection{Comparison with Existing Algorithms}

We compare the performances among three scheduling schemes: 1) our proposed scheduling scheme, 2) a Large-Metric-First scheduling scheme [8], and 3) the Guaranteed Bit Rate (GBR)/Non-GBR scheduling scheme [15]. The Large-Metric-First scheduling schemeis determined by a utility function for UEs, which is given by $\lambda=\mathrm{W}_{\mathrm{P}}+\mathrm{P}_{\mathrm{PF}}$, where $\mathrm{W}_{\mathrm{P}}$ is the weight for UEs in the smart grid communication network and $\mathrm{P}_{\mathrm{PF}}$ is given by traditional 
LTE scheduling proportional fair (PF) algorithm. The GBR/Non-GBR scheduling represents a guaranteed minimum bit rate requested by an application. In LTE, the GBR bearers and non-GBR bearers can be provided. Of these, the GBR bearers are typically used for applications such as exception messages and control messages, with an associated GBR value; higher bit rates can be allowed if resources are available. Non-GBR bearers do not guarantee any particular bit rate, which usually are used for the normal operation applications. All simulations have been conducted with the parameters described in Section 3.4.3.

\subsubsection{Simulation Results}

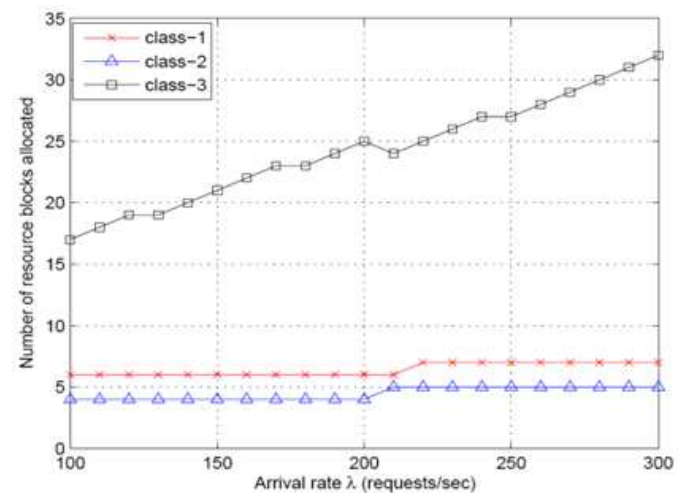

Figure 3.Number of resource blocks for different classes in the proposed scheduling scheme
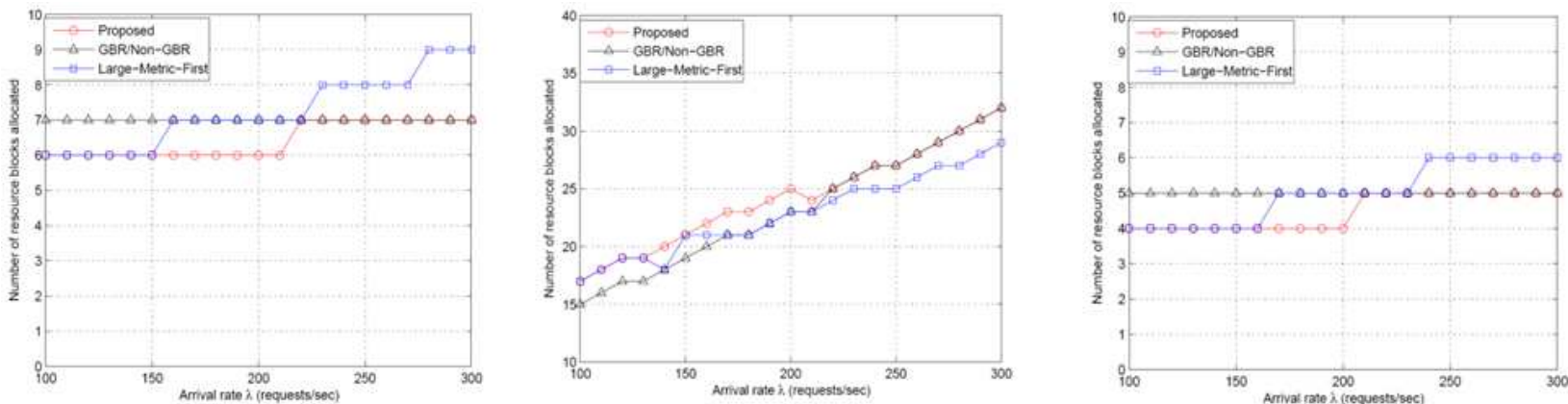

Figure 5. The number of allocated resource blocks for different classes
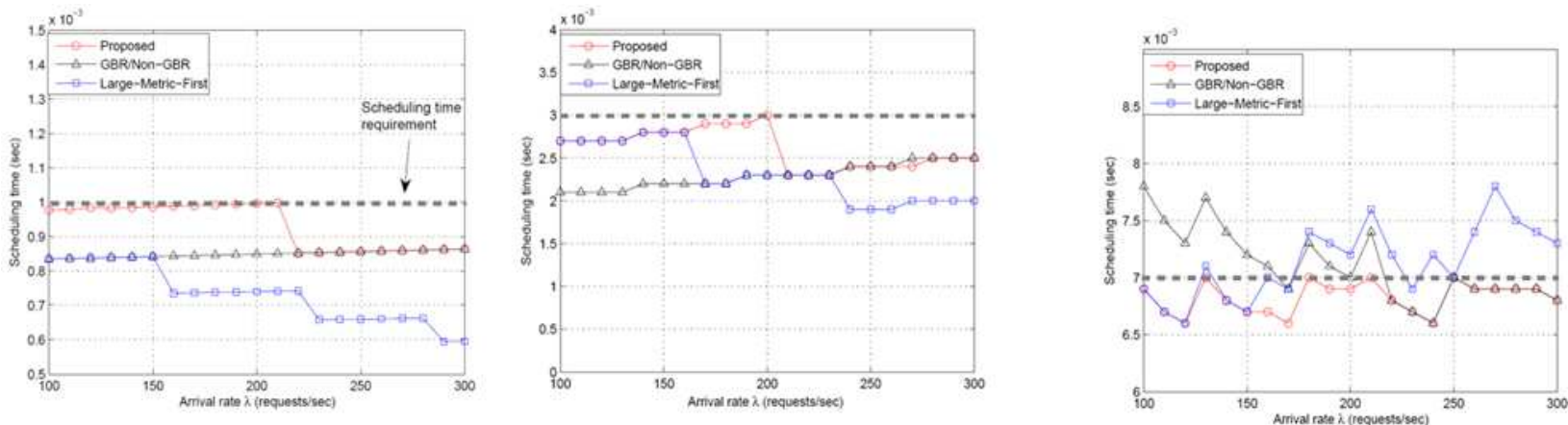

Figure 6.The scheduling time for different classes

Fig. 5 shows the number of allocated resource blocks for different classes among the three scheduling schemes. The total available resource blocks for an arrival rate are the same among the three schemes. For example, when $\lambda=150$ request/s, our proposed algorithm uses 31 resource blocks, then the other two algorithms also have 31 resource blocks 
available in the same arrival rate. Fig.5(a), Fig.5(b) and Fig.5(c) show the number of resource blocks for three classes, respectively. We can see that more resource blocks are assigned for class- 1 and class- 2 in Large Metric-First algorithm than our proposed algorithm. That is because the Large-Metric-First scheduling scheme is more focused on the traffic with higher priorities. In the GBR/Non-GBR scheduling scheme, class-1 and class-2 traffic flowsare assigned to GBR bearers and the values do not dynamically change with the increasing of arrival rate.

We can see that such kind of scheduling is inflexible. If the smart grid system encounters an emergency situation, for example, the additional volume of exceptional messages, alarms and control trafficare added in the networks, the scheduling time performance will get much worse.

Fig. 6 shows the scheduling time for different classes using the same amount of resource blocks indicated in Fig.4. The grey dash lines represent the scheduling time requirements for different classes (see Table 1). The Large-Metric-First scheduling and GBR/Non-GBR scheduling allocates more resource blocks in class- 1 and class-2, and less resource blocks in class-3. All these three algorithms satisfy the scheduling time requirements for class- 1 and class, but for class- 3 traffic flow, the other two algorithms cannot satisfy the requirement because less resource blocks are left for class-3. The Large-Metric-First scheduling has a better performance in class- 1 and class- 2 while sacrifices the scheduling time in class-3. The GBR/Non-GBR can partly satisfy the requirement for class-3 when arrival rate is larger than 220 requests/sec.

\section{Conclusion}

In this paper, we proposed an optimal scheduling algorithm for LTE in smart grid and evaluated the scheduling time performance in the smart grid network environment. The experiment results show that the scheduling time in the proposed scheme outperforms Large-Metric-First scheduling scheme and GBR/Non-GBR scheduling scheme.

\section{References}

[1] L. B. Le and T. Le-Ngoc, "QoS provisioning for OPFMA-based wireless network infrastructure in smart grid," in proc. of IEEE Electrical and Computer Engineering 2011 24th Canadian Conference on CCECE, Niagara Falls, May 2011.

[2] Y. Kim and M. Thottan, "SGTP: Smart Grid Transport Protocol for secure delivery periodic real time data," Bell Labs Technical Journal, vol. 16, no. 3, pp. 83-99, December 2011.
[3] O. C. Onar, M. Starke, G. P. Andrews and R. Jackson, "Modeling, controls, and applications of community energy storage systems with used EV/PHEV batteries," in proc. of IEEE Transportation Electrification Conference and Expo (ITEC), Dearborn, June 2012.

[4] B. P. Roberts and C. Sandberg, "The role of energy storage in development of smart grids," in Proceedings of the IEEE, vol. 99, no. 6, pp. 1139-1144, June 2011.

[5] P. Cheng, L. Wang, B. Zhen and S. Wang, "Feasibility study of applying LTE to smart grid," in proc. ofSmart Grid Modeling and Simulation (SGMS), 2011 IEEE First International Workshop, Brussels, Oct 2011.

[6] S. Abeta, "Toward LTE commercial launch and future plan for LTE Enhancements (LTE-Advanced)," in proc. of Communication Systems (ICCS), 2010 IEEE International Conference, Singapore, Nov 2010.

[7] A. Ghosh and R. Ratasuk, Essentials of LTE and LTE-A, Cambridge University Press, 2011.

[8] Y. Xu and C. Fischione, "Real-time scheduling in LTE for smart grids," in proc.of IEEE Communications Control and Signal Processing (ISCCSP), 2012 5th International Symposium, Rome, May 2012.

[9] S. N. K. Marwat, T. Weerawardane, Y. Zaki, C. Goerg and A. Timm-Giel, "Performance evaluation of bandwidth and QoS aware LTE uplink scheduler," in 10th International Conference, WWIC 2012, Santorini, Greece, June 6-8, 2012. Proceedings, June 2012.

[10] O. Delgado and B. Jaumard, "Scheduling and resource allocation in LTE uplink with a delay requirement," in proc. or IEEECommunication Networks and Services Research Conference (CNSR), 2010 Eighth Annual, Montreal, May 2010 .

[11] D. Gross and C. M. Harris, Fundamentals of queuing theory, New York: Wiley, 1998.

[12] M. M. Tantawy, A. S. T. Eldien and R. M. Zaki, "A novel cross-layer scheduling algorithm for Long Term-Evolution (LTE) wireless system," Canadian Journal on Multimedia and Wireless Networks, vol. 2, no. 4, pp. 57-62, December 2011.

[13] "ETSI TR 136 942; LTE; evolved universal terrestrial radio access(E-UTRA); radio frequency (RF) system scenarios (3GPP TR 36.942 version 9.3.0 Release 9)," 3GPP, 2012

[14] .H. Safa and K. Tohme, "LTE uplink scheduling algorithms: Performance and Challenges," in proc. of IEEETelecommunications (ICT), 2012 19th International Conference, Jounieh, April 2012.

[15] M. Alasti, B. Neekzad, J. Hui and R. Vannithamby, "Quality of service in WiMAX and LTE networks [Topics in Wireless Communications]," Communications Magazine, IEEE, vol. 48, no. 5, pp. 104-111, May 2010. 